\title{
A Design Method of Block Interleaver for Turbo Codes Based on Low Correlation Coefficient
}

\author{
Student Member Xiaoqiu Wang (Chiba University) \\ Student Member Hua Lin (Chiba University) \\ Member Jianming Lu (Chiba University) \\ Non-member Takashi Yahagi (Chiba University)
}

Turbo codes, also known as parallel concatenated convolutional codes (PCCC), were demonstrated to have performance within $0.5 \mathrm{~dB}$ of the Shannon limit around $\mathrm{BER}=10^{-5}$ over AWGN channel. However, these simulation results were only based on large interleaver size and many decoding iterations. In personal communication systems, the standard rate of tone is $9.6 \mathrm{kbit} / \mathrm{s}$ and the time interval of each frame is $20 \mathrm{~ms}$. So the length of a transmission frame is about 192 bits and is much shorter than the $256 \times 256$ frame. When the short frame transmission is taken into consideration, a proper interleaver design is very important. In this letter, the theoretical analysis of the performance of an interleaver based on the correlation coefficient is presented. An "inverse diagonal" interleaver is proposed to show that it has the lower correlation coefficient compared with the conventional row-column block interleaver design and is easily realized.

Keywords: Turbo codes, block interleaver, correlation coefficient

\section{Introduction}

The structure of turbo encoder is the parallel concatenation of two recursive systematic convolutional encoders (RSC) that make parity symbols using input bit frame with an interleaver in-between (1),(2). The coding rate will be punctured to $1 / 2$ by alternatively selecting bits from two RSCs. The turbo decoder consists of two concatenated decoders of the component codes separated by the same interleaver.

The basic role of the interleaver is that it can spreads out burst errors. The interleaver provides "scrambled" information data to the second component encoder and decorrelates the inputs to the two component decoders so that an iterative decoding algorithm based on "uncorrelated" information exchange between the two component decoders can be realized ${ }^{(3)}$. For example, after correction of some of the errors in the first component decoder, some of the remaining errors can be spread by the interleaver so that they become correctable in the other decoder. By increasing the number of iterations in the decoding process, the bit error probability approaches the channel capacity ${ }^{(4)}$.

When turbo code is applied in short frame transmission systems ${ }^{(5)}$, a proper interleaver design is very important. In this letter, the theoretical analysis of the performance of an interleaver based on the correlation coefficient is presented. An "inverse diagonal" block interleaver is proposed to show that it has the lower correlation coefficient compared with the conventional row-column block interleaver design and is easily realized.

2. The Proposed "Inverse Diagonal" Block Interleaver

To analyze the correlation characteristic of an inter- leaver, we first give the definition of the correlation coefficient of two data sequences.

Definition: The average correlation coefficient of data sequence $X=\left\{x_{1}, x_{2}, x_{3}, \cdots \cdots, x_{n}\right\}$ and $Y=$ $\left\{y_{1}, y_{2}, y_{3}, \cdots \cdots, y_{n}\right\}$ (the value of $x_{i}$ and $y_{i}$ is 0 or 1 ) is

$$
R_{X Y}=\frac{1}{N} \sum_{i=1}^{N}\left(2 x_{i}-1\right)\left(2 y_{i}-1\right) \cdots \cdots \cdots(1)
$$

From the analysis in Section 1, we know that an interleaver can be realized by decorrelating its inputs and outputs. A conventional block interleaver is a memory in which data is mapped onto an $m \times n$ matrix in a row-by-row fashion and read out in a column-by-column fashion. In this letter, we will propose an inverse diagonal interleaver based on low correlation characteristic. This interleaver is a memory in which data is written into an $m \times n$ matrix in a row-by-row fashion and read out in an inverse diagonal fashion.

Figure 1 is a $4 \times 4$ interleaver example. The input sequence is $a_{11} a_{12} a_{13} a_{14} a_{21} a_{22} \cdots a_{44}$, and the output of the interleaver is $a_{44} a_{34} a_{43} a_{24} a_{33} a_{42} \cdots a_{11}$. If the length of an interleaver is $m \times n$ ( $\mathrm{m}$ is the rows of the interleaver and $n$ is the columns of the interleaver), the input information sequence and the output sequence of the interleaver are expressed as $\left\{s_{1}^{I}, s_{2}^{I}, s_{3}^{I} \cdots s_{m \times n}^{I}\right\}$ and $\left\{s_{1}^{O}, s_{2}^{O}, \cdots, s_{m \times n}^{O}\right\}$ respectively. Then the average correlation coefficient between the input sequence and the output sequence can be defined by

$$
R_{I}=\frac{\sum_{i=1}^{m \times n}\left(2 s_{i}^{I}-1\right)\left(2 s_{i}^{O}-1\right)}{m \times n}
$$

where $s_{i}^{I}$ and $s_{i}^{O}$ are chosen from the binary signal set 


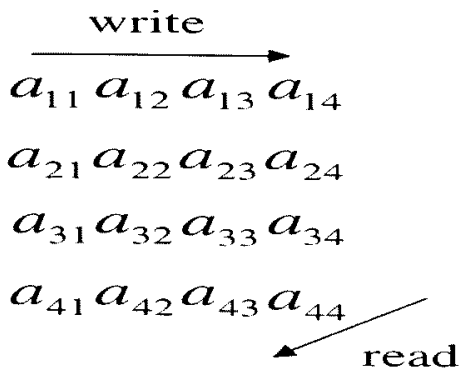

Fig. 1. An example of the proposed interleaver

Table 1. Theoretical correlation coefficients of different interleavers

\begin{tabular}{|l|c|c|c|}
\hline Interleaver model & $12 \times 16$ & $13 \times 15$ & $14 \times 14$ \\
\hline Fixed points of $I_{0}$ & 2 & 3 & 14 \\
\hline Fixed positions of $I_{1}$ & 0 & 1 & 2 \\
\hline$R_{I_{0}}$ & 0.0104 & 0.0154 & 0.0714 \\
\hline$R_{I_{1}}$ & 0 & 0.0051 & 0.0102 \\
\hline
\end{tabular}

$\{0,1\}$. In theoretical analysis, the probability of the distribution of the transmitted signal is assumed to be equal. It means that $P\left(s_{i}^{I}=1\right)=P\left(s_{i}^{I}=0\right)=1 / 2$ and $P\left(s_{i}^{O}=0\right)=P\left(s_{i}^{O}=1\right)=1 / 2($ for $i=1,2, \cdots, m \times n)$. From the statistics points of view, the correlation coefficient is equal to 0 if the input sequence and output sequence are statistically independent. The calculation of the correlation coefficient of the interleaver will be estimated by the concept of statistical average. That is, it is decided by the fixed positions in the interleaving algorithm. This means that some positions of the input sequence are unchanged when passing through the interleaver. At the fixed point $f, s_{f}^{I}=s_{f}^{O}$, then we have

$$
\left(2 s_{f}^{I}-1\right)\left(2 s_{f}^{O}-1\right)=\left(2 s_{f}^{I}-1\right)^{2}=1 \cdots \cdots \cdot
$$

In other place, the probability of $\left(2 s_{i}^{I}-1\right)\left(2 s_{i}^{O}-1\right)$ can be described by

$$
\begin{aligned}
& P\left\{\left(2 s_{i}^{I}-1\right)\left(2 s_{i}^{O}-1\right)=1\right\} \\
= & P\left\{\left(2 s_{i}^{I}-1\right)\left(2 s_{i}^{O}-1\right)=-1\right\}=1 / 2
\end{aligned}
$$

Therefore, the average correlation coefficient can be approximately estimated by the concept of fixed point as mentioned above.

$$
R_{I}=\frac{\text { Fixed points of an interleaver }}{m \times n} \ldots \ldots
$$

The fixed positions can be found by computer searching. Table 1 is the comparison of the correlation coefficient of the proposed interleaver and the conventional "row-column" interleaver based on the concept of fixed positions when the information sequence passes through the interleaver. In Table $1, I_{0}$ and $I_{1}$ represent the conventional "row-column" interleaver and the "inverse diagonal" interleaver, respectively. The data frame length is set to be about 192 bits. From Table 1, we find that the proposed "inverse diagonal" interleaver has lower correlation coefficient than the conventional "row-column" interleaver, especially when the input sequence is a square matrix $(m=n)$.

電学論C, 122 巻 4 号, 平成 14 年
Table 2. Simulation results of correlation coefficients of different interleavers

\begin{tabular}{|l|c|c|c|}
\hline $\mathrm{R}$ & Test 1 & Test 2 & Test 3 \\
\hline$R_{I_{0}}(12 \times 16)$ & 0.0089 & 0.0103 & 0.0121 \\
\hline$R_{I_{1}}(12 \times 16)$ & 0.0004 & 0.0002 & 0.0003 \\
\hline$R_{I_{0}}(13 \times 15)$ & 0.0163 & 0.0148 & 0.0150 \\
\hline$R_{I_{1}}(13 \times 15)$ & 0.0051 & 0.0054 & 0.0061 \\
\hline$R_{I_{0}}(14 \times 14)$ & 0.0692 & 0.0704 & 0.0726 \\
\hline$R_{I_{1}}(14 \times 14)$ & 0.0111 & 0.0096 & 0.0120 \\
\hline
\end{tabular}

\section{Simulations and Results Analysis}

To prove the theoretical analysis mentioned above, we calculate the correlation coefficient of an interleaver by computer simulation. The selected binary signal generator is Gaussian model. Because the signal generator has a random characteristic, the probability distributions of 0 and 1 may not be strictly equal at some frames, but the Gaussian model is approximate to the real transmitted signal. Therefore, the reliability of the simulation results can be accepted. In our simulation, the number of transmitted signals is about 30000 . Table 2 is the simulation results of different interleavers. For each interleaver, we try 3 tests. From Table 2, we find that the results of correlation coefficient are approximately equal to the results by the concept of fixed positions mentioned above though there is slight deviation from the theoretical results. The deviation is caused by unequal probability distribution of 0 and 1 in real transmission systems and it will be mitigated if the number of transmitted signals are large enough. Moreover, the proposed "inverse diagonal" interleaver shows the lower correlation coefficient than the "row-column" interleaver.

\section{Conclusions}

In this letter, we have discussed the interleaver design method in turbo codes system based on low correlation coefficient when short frame transmission is taken into consideration. The calculation of correlation coefficient of a block interleaver is simplified by calculating the fixed positions of the interleaver according to the statistical average analysis. Theoretical analysis and simulation results have shown that the proposed "inverse diagonal" block interleaver has better performance than the conventional "row-column" block interleaver.

(Manuscript received July 23, 2001)

\section{References}

(1) C. Berrou, A. Glavieux and P. Thitimajshima, "Near Shannon limit error correcting coding and decoding: turbo-codes (1), "IEEE ICC'93, pp.1064-1070, 1993.

(2) C. Berrou and A. Glavieux, "Near optimum error correcting coding and decoding: turbo-codes," IEEE Trans. Commun., vol.44, No.10, pp.1261-1271

(3) B. Vucetic and J. Yuan, Turbo Codes, Principles and Applications, Kluwer Academic Publishers, 2000.

(4) C. Heegard and S.B. Wicker, Turbo Coding, Kluwer Academic Publishers, 1999.

(5) P. Jung and M. Nabhan, "Performance evaluation of turbocodes for short frame transmission system," Electron. Lett. vol.30, pp.111-113, 1994 